\title{
Male involvement in birth preparedness and complication readiness for emergency referral at Sodo town of Wolaita zone, South Ethiopia: a cross sectional study
}

\author{
Kebreab Paulos ${ }^{1 *}$ (D) Nefsu Awoke², Bazie Mekonnen ${ }^{3}$ and Aseb Arba²
}

\begin{abstract}
Background: Preventable maternal mortality remains a huge burden more especially in sub-Saharan Africa. The involvement of male partner during pregnancy and its complication helps an expectant mother to make timely decisions to avoid delays that brings about complications that could result in morbidity or mortality.

Methods: Institution based cross sectional study was conducted in 2017, at Sodo Town of Wolaita Zone among mothers who came to hospital and admitted to $\mathrm{MCH}$ department due to emergency obstetric referral. Data were collected using pre-tested and structured questionnaire. The collected data entered by Epi data, cleaned and analyzed by using SPSS for windows version 23.0. A descriptive analysis was done using frequency, mean, quartile and standard deviation. Bivariate and multivariable logistic regression was carried out to identify the associated factors. Level of statistical significance was declared at $p$ value $<0.05$. Finally the results of Bivariate and multivariable logistic regression analysis were presented in crude and adjusted odds ratio with $95 \%$ confidence intervals.

Result: Data were obtained from 233 women, with a response rate of 100\%. The prevalence of male partner's involvement in birth preparedness and complication redness for emergency referral in this study was 30.9\%. After adjusting for the effect of confounding variables using multivariable logistic regression, variables like distance of health facility ( $A O R=0.29,95 \% \mathrm{Cl}=0.12,0.72$ ), having $A N C$ follow-up $(A O R=2.9,95 \% \mathrm{Cl}=1.52-5.51)$ and experience of obstetric complication ( $A O R=1.79,95 \% \mathrm{Cl}=1.06-3.04$ ) have statistically significant association with male partner's involvement in birth preparedness and complication readiness for obstetric referral.

Conclusion: In general, male partner's involvement in birth preparedness and complication readiness for obstetric referral in the study area was low. Antenatal care attending and experiencing of obstetric complication were factors determining male partner's involvement in complication readiness. Health care professionals should involve male partners to attend ANC clinic at each stage and arrange special antenatal care conferences which may increase awareness and practice about complication readiness and plan.
\end{abstract}

Keywords: Male involvement, Birth, Obstetric complications, Emergency referral, Wolaita zone, Sodo town

\footnotetext{
* Correspondence: kebreabpaulos@gmail.com

${ }^{1}$ Department of Midwifery, College of Health and Medical Sciences, Wolaita

Sodo University, Wolaita Sodo, Ethiopia

Full list of author information is available at the end of the article
}

(c) The Author(s). 2020 Open Access This article is distributed under the terms of the Creative Commons Attribution 4.0 International License (http://creativecommons.org/licenses/by/4.0/), which permits unrestricted use, distribution, and reproduction in any medium, provided you give appropriate credit to the original author(s) and the source, provide a link to the Creative Commons license, and indicate if changes were made. The Creative Commons Public Domain Dedication waiver (http://creativecommons.org/publicdomain/zero/1.0/) applies to the data made available in this article, unless otherwise stated. 


\section{Background}

Maternity carries risk on life of women due to obstetric and emergent complication. Maternal deaths in developing countries share almost all of maternal death of the world, which accounts for more than a million of all deaths [1]. World Health Organization (WHO) reported that maternal death in developing countries accounted for 286,000 of all maternal deaths as a consequence of preventable complications. These deaths are caused from prenatal period complications [2].

In our country Ethiopia, the ratio of maternal mortality (MMR) is estimated to be 412 deaths per 100,000 live births according to the report from Ethiopian Demographic Health Survey (EDHS) [3]. In our country Ethiopia, the ratio of maternal mortality (MMR) is estimated to be 412 deaths per 100,000 live births according to the report from Ethiopian Demographic Health Survey (EDHS) [3]. It was declined from 1400 in 1990 to 412 in 2015 by $71 \%$ which was subsequently near the objective for MDG 5 (75\%) [4].

Regardless of these general accomplishments in the health related MDGs in Ethiopia, there were additionally difficulties that would likewise be worries during the time of the SDGs. Government expense on health is $4.9 \%$ which is still underneath the Abuja responsibility to burn through $15 \%$ of the administration spending plan on health. Per capita health utilization was underneath 2001 WHO recommendation to be US\$35 however it was US\$26.7 in 2014 [4]. To tackle this Ethiopia has adapted the current global development agenda (SDGs) which has 17 goals, with Goal 3 focusing on health (ensure healthy lives and promote well-being for all at all ages) [5].

A main strategy to reduce maternal mortality and morbidity from complications is equipping family with awareness of birth plan that include birthpreparedness and complication readiness procedures specially for pregnant women, their husbands and their families [6]. Birth preparedness and complication readiness $(\mathrm{BP} / \mathrm{CR})$ is a wide-ranging and inclusive package aimed at encouraging timely access to skilled maternal and neonatal services, promotes active preparation and decision making for delivery by pregnant women and their families [7].

The most important components of birth plan package include recognition of danger sign, a plan for birth attendant, a plan for place of delivery, saving money for transport or other costs in case the need arise. In sub-Saharan Africa, pregnancy and childbirth remain to be viewed merely as women issues [8]. A male companion during perinatal care is unusual and rare in many communities, it is unthinkable to find male partners accompanying a woman to the labor room during delivering [9].
Nevertheless, men have social and economic power, especially in Africa, and have significant control over their partner. They decide the timing and condition of sexual relation, family size, whether their partner will utilize available maternal health care [10]. Hence this condition makes male partners participation critical if improvement in maternal health and reduction of maternal morbidity and mortality is to be realized. Strategies for enhancing involvement of men in maternal health services should aim at raising their awareness about emergency obstetrics conditions, and engaging them in birth preparedness and complication readiness [11].

Maternal mortality and morbidity associated to pregnancy and childbirth could be prevented if women and their families identify when and where to seek help, have access to the healthcare system during pregnancy, childbirth and the postpartum period and subsequently receive care from skilled provide [12].

Lack of husband participation in birth preparedness plans and delays in care seeking for obstetric emergencies are main contributing factor of maternal death. Male involvement is essential for improving birth preparedness because of patriarchy which agree to men to control women's access to and utilization of maternal health care and safe motherhood programs which may be affected by male partner participation because husbands were the most influential decision-maker and as the key member of the family [13].

Despite the great ability of Birth Preparedness and Complication Readiness in reducing the maternal and newborn deaths, its importance is not well known in most of sub-Saharan Africa. Therefore this study aimed to assess male involvement in birth preparedness and complication readiness for emergency referral.

\section{Methods}

Study area

The study was conducted in Sodo Christian General Hospital (SCGH) and Wolaita Sodo University Teaching and Referral Hospital (WSUTRH) which is located in Sodo town, Wolaita Zone. Wolaita Sodo is main town of Wolaita Zone, Southern Nations, Nationalities, and Peoples Region (SNNPR) of Ethiopia. Wolaita Sodo is located $332 \mathrm{~km}$ south of Addis Ababa and $122 \mathrm{~km}$ south of Hawassa. Sodo Christian General Hospital is only private hospital in Sodo Town and WSUTRH, former Otona Hospital is recently held by Wolaita Sodo University. These hospitals serve population of Wolaita Zone and surrounding zones and regions of Ethiopia as referral hospital.

\section{Study design and period}

A cross-sectional study design was conducted at Sodo Christian general hospital and Wolaita Sodo university 
teaching and referral hospital maternity ward from July 01 to October 30, 2017.

\section{Study population}

All mothers who were admitted at maternity unit of two hospitals due to obstetric emergency were source population and mothers who are admitted to maternity unit due to obstetric emergency during the period of data collection were considered as study population. The study inclusion criteria were having been admitted to the hospital as an emergency referral in antenatal, labor or the postpartum period and willingness to consent for participation in the study. Participants were followed up to their discharge from hospitals or death.

\section{Sample size and sampling technique}

Sample size was determined by using single population proportion formula based on the following assumptions: 95\% confidence level, prevalence of male involvement in birth preparedness and complication readiness is $60.4 \%$ from previous study [14], and a 5\% margin of error. The Calculated sample size was $n=368$.

The last quarter (3 months) data of the two hospitals show that in total of 500 (292 in WSUTRH and 208 SCGH) mothers were admitted to maternity unit due to obstetric complications. Since total population of this study less than 10,000 we used correction formula.

$$
n=\frac{n}{1+\frac{n}{N}}=\frac{368}{1+\frac{368}{500}}
$$

Expecting a $10 \%$ non-response rate the final sample size calculated was 233 .

The total sample size was allocated proportionally to each hospital by reviewing the numbers of mothers who were admitted to maternity unit due to obstetric complications in the last quarter of 2016. Systematic random sampling technique was utilized to approach the study participant who fulfills the inclusion criteria. The lists of mothers were obtained from admission registry of maternity unit, and this was used to draw sample of the study participant. Calculated Kth value is 2 and first random start 1 chosen by lottery method and data were collected every 2 nd interval.

\section{Data collection tool}

Interviewer administered questionnaires were conducted for the quantitative study. The questionnaire was adapted from the survey tools developed by JHPIEGO Maternal and Neonatal Health Program [15]. It is divided into four parts. The first section inquired about personal data, including age, occupation, ethnicity, religion and educational level. The second part elicited information about Knowledge of obstetric complication and Sours of information. The third section assessed birth preparedness and complication readiness and health seeking behaviors.

Male partner involvement: refers to its involvement in joint decisions on where to attend ANC, where to deliver, save money for emergency, knowing and deciding earlier on where to go during emergency case.

\section{Data collection process}

Four experienced female data collectors were collected the data after thorough training on the objective of the study and the questionnaire. Two degree holder health professionals supervised the data collectors. Data collectors and supervisors were trained for 5 days by using training manual prepared for this purpose. Data collection was conducted after women managed for the emergency case and willingness to respond to the questions.

\section{Data analysis}

The collected data coded and entered by Epi data version 3.1 and cleaning and analyzed by SPSS version 23.0. A descriptive analysis was done using frequency, mean, quartile and standard deviation. Bivariate logistic regression was carried out to identify the associated factors. All variables with $p$ value $\leq 0.25$ were taken into consideration in the multivariable model to control for all possible confounders and the variables were selected by enter method to see the efect of each variable on the outcome variable. Multicollinearity tests were carried out to see the correlation between independent variables using standard error and one of the independent variable was dropped for those with standard error of $>2$. Finally the results of multivariable logistic regression analysis were presented in crude and adjusted odds ratio with 95\% confidence intervals. Level of statistical significance was declared at $p$ value $<0.05$.

\section{Data quality control}

To maintain the validity of the data collection tool the questionnaire was first translated to Amharic then to Wolaitigna and retranslated to English after data collection to maintain consistency by native language translator. Training on data collection and procedure was provided to data collectors and supervisors. Pretest was conducted on $5 \%$ of sample size in Areka town Dubo hospital.

\section{Ethical considerations}

Ethical clearance letter was obtained from Addis Ababa University Department of Nursing and Midwifery research committee and College of Health Sciences Institutional Review Board. Written permission was requested from Wolaita Sodo zone health bureau. Data collectors given consent to collect right data from 
participants and to respect rights of participants. Moreover, all the study participants were informed verbally about the purpose and benefit of the study along with their right to refuse and verbal consent was obtained. Furthermore, the study participants are reassured for an attainment of confidentiality and written consent information is also taken.

\section{Results}

\section{Socio-demographic characteristics of respondents and} male partner

A total of 233 Married women who had come to hospital due to emergency obstetric referral were interviewed with response rate of $100 \%$. Majority of the women were in the age group of 25 and above (72.5\%) with mean age of $26.7 \pm 4.8$ standard deviation, while most of male partners were in the age group of 30 and above years of age (73\%) with mean age of $31.9 \pm 4.9$ standard deviation. Regarding educational level (33.5\%) women and $36.5 \%$ of male partners had primary level of education. Most of the women were housewives $(47.5 \%)$ whereas $(38.3 \%)$ of male partners were Gov. Organization employed (Table 1) both women and male partners were similar in their religion.

\section{Birth preparedness and complication readiness plan}

Majority 214(91.8\%) of the respondents had a plan for delivery. Ten (4.3\%) of the women delivered at home previously from those women $4(1.7 \%)$ mentioned distance from health facility is too far as reason for home delivery. Midwifes/nurse assisted $119(51.1 \%)$ of women delivery. Twenty one (9\%) of women experienced problem during pregnancy of them 13(5.6\%) experienced bleeding during pregnancy, $16(6.9 \%)$ seek health care due to the problem (Table 2).

\section{Level of male partners involvement on decision for} antenatal care follow-up

Majority 225(96.6\%) of the women received ANC at least once and the rest $8(3.4 \%)$ do not attended ANC service. One hundred eight seven (80.5\%) of women and their male partner both decided to follow ANC (Fig. 1).

\section{Reason for referral male partners accompanied to place of delivery}

Among the reason for referral 69(29.6\%) were referred due to obstructed or prolonged labor followed by 34(14.6\%) fetal distress, 27(11.6\%) Malpresentation, 20(8.6\%) Previous caesarean section, 16(6.9\%) Multiple pregnancy, 16(6.9\%) Postpartum hemorrhage, 16(6.9\%) Anemia, 14(6\%) hemorrhage, 13(5.6\%) Poor obstetric history, 10(4.3\%) Ruptured uterus, 9(3.9\%) Eclampsia, 7(3\%) Preeclampsia, and
Table 1 Socio-demographic characteristics of the Women and Male partners in Wolaita zone Sodo town, 2017

\begin{tabular}{|c|c|c|}
\hline Variables $(n=233)$ & Category & n (\%) \\
\hline \multirow[t]{2}{*}{ Women age (Years) } & $<25$ & $64(27.1)$ \\
\hline & $\geq 25$ & $\begin{array}{l}169 \\
(72.5)\end{array}$ \\
\hline \multirow[t]{2}{*}{ Male partners Age (Years) } & $<30$ & $63(27)$ \\
\hline & $\geq 30$ & $170(73)$ \\
\hline \multirow[t]{4}{*}{ Women Educational Level } & No Formal Education & $50(21.5)$ \\
\hline & Primary (1-8) & $78(33.5)$ \\
\hline & Secondary (9-12) & $53(22.7)$ \\
\hline & Tertiary (above 12) & $52(22.3)$ \\
\hline \multirow{4}{*}{$\begin{array}{l}\text { Male partners Educational } \\
\text { Level }\end{array}$} & No Formal Education & $19(8.2)$ \\
\hline & Primary (1-8) & $85(36.5)$ \\
\hline & Secondary (9-12) & $50(21.5)$ \\
\hline & Tertiary (above 12) & $79(33.9)$ \\
\hline \multirow[t]{6}{*}{ Women Occupation } & Gov.Org.Employed & $45(19.3)$ \\
\hline & $\begin{array}{l}\text { NGO or Private Org. } \\
\text { Employed }\end{array}$ & $3(1.3)$ \\
\hline & Merchant & $49(21)$ \\
\hline & Daily Laborer & $7(3)$ \\
\hline & Housewife & $\begin{array}{l}111 \\
(47.5)\end{array}$ \\
\hline & Other & $18(7.7)$ \\
\hline \multirow[t]{6}{*}{ Male partners Occupation } & Gov.Org.Employed & $66(28.3)$ \\
\hline & $\begin{array}{l}\text { NGO or Private Org. } \\
\text { Employed }\end{array}$ & $14(6)$ \\
\hline & Merchant & $63(27)$ \\
\hline & Daily Laborer & $38(16.3)$ \\
\hline & Farmer & $51(21.9)$ \\
\hline & Other & $1(0.4)$ \\
\hline
\end{tabular}

5(2.1\%) Preterm labour. From the women referred to hospital majority $189(81.1 \%)$ reached hospital by ambulance. Among women referred to hospital 75(32.2\%) were accompanied to deliver place by male partner and majority $156(67 \%)$ of women were accompanied by other member of the family member. Majority $164(70.4 \%)$ of the women get health facility in distance of greater than $5 \mathrm{~km}$ (Table 3).

Factors associated with male partner involvement during birth preparedness and complication readiness for obstetric referral

There were seven variables in binary logistic regression which had a $p$-value of $\leq 0.25$ and became a candidate for multiple logistic regressions. After adjusting for the effect of confounding variables using Multiple logistic regression, three variables like distance of health facility ( $\mathrm{AOR}=0.29,95 \% \mathrm{CI}=0.12,0.72$ ), having ANC follow-up $(\mathrm{AOR}=2.9,95 \% \mathrm{CI}=1.52-5.51)$ and 
Table 2 Birth preparedness and health seeking behaviors at Sodo town in Wolaita zone, 2017

\begin{tabular}{|c|c|c|}
\hline Variables $(n=233)$ & Category & n (\%) \\
\hline \multirow[t]{2}{*}{ Made a plan for deliver } & Yes & $\begin{array}{l}214 \\
(91.8)\end{array}$ \\
\hline & No & $\begin{array}{l}19 \\
(8.2)\end{array}$ \\
\hline \multirow[t]{2}{*}{ Place of previous delivery } & Health facility & $\begin{array}{l}223 \\
(95.7)\end{array}$ \\
\hline & Home & $\begin{array}{l}10 \\
(4.3)\end{array}$ \\
\hline \multirow[t]{4}{*}{ Reason for home delivery $(n=10)$} & $\begin{array}{l}\text { Far distance from } \\
\text { health facility }\end{array}$ & $4(1.7)$ \\
\hline & No transportation & $3(1.3)$ \\
\hline & Cost too much & $2(0.9)$ \\
\hline & Delay to seek care & $1(0.4)$ \\
\hline \multirow[t]{4}{*}{ Delivery assisted by } & Midwife or Nurse & $\begin{array}{l}119 \\
(51.1)\end{array}$ \\
\hline & Doctor & $\begin{array}{l}100 \\
(42.9)\end{array}$ \\
\hline & HEW & $7(3)$ \\
\hline & Others & $7(3)$ \\
\hline \multirow{2}{*}{$\begin{array}{l}\text { Experienced problem during } \\
\text { pregnancy }\end{array}$} & Yes & $21(9)$ \\
\hline & No & $\begin{array}{l}212 \\
(91)\end{array}$ \\
\hline \multirow[t]{4}{*}{ Problems experienced } & Bleedings & $\begin{array}{l}13 \\
(5.6)\end{array}$ \\
\hline & Loss of consciousness & $3(1.3)$ \\
\hline & Severe headache & $2(0.9)$ \\
\hline & Other & $5(2.1)$ \\
\hline \multirow[t]{2}{*}{$\begin{array}{l}\text { Seek health care during problem and } \\
\text { complications }\end{array}$} & Yes & $\begin{array}{l}16 \\
(6.9)\end{array}$ \\
\hline & No & $5(2.1)$ \\
\hline
\end{tabular}

\section{Discussion}

The aim of this study was to assess male partners' involvement in in birth preparedness and complication readiness for obstetric referral of a spouse and its associated factors among women admitted at $\mathrm{MCH}$ department due to obstetric referral at Sodo town in Wolaita zone, Southern Ethiopia.

In this study prevalence of male partners' involvements' in birth preparedness and complication readiness for obstetric referral was $30.9 \%$. Women who had any experience of pregnancy complications were 1.79 times and those who received ANC follow up at list one time were 2.9 times more likely to involve male in birth preparedness and complication readiness for obstetric referral whereas women's who walked less than $5 \mathrm{~km}$ distance to the nearest health facility had lower odds of male partners involvement in birth preparedness and complication readiness for obstetric referral compared to those who walked more than $5 \mathrm{~km}$.

Having ANC follow-up of women at list once was significant predictor for male partners' involvement in birth preparedness and complication readiness for obstetric referral. Respondent who had ANC follow-up at list once were 2.9 times more likely male involve in birth preparedness and complication readiness for obstetric referral than those had no ANC follow up. The finding was consistent with other study done in Haiti, Ambo and Addis Ababa $[9,16,17]$. Having ANC follow-up at list once could influence male partner's involvement in




Table 3 Male partner involvement in decision making for health care seeking at Sodo town in Wolaita zone, 2017

\begin{tabular}{lll}
\hline Variables $(n=233)$ & Category & $\mathrm{n}(\%)$ \\
\hline Means of transportation & Ambulance & 189 \\
& & $(81.1)$ \\
& Privet car & $23(9.9)$ \\
& Taxi/bus & $21(0.9)$ \\
Who accompanied to place of & Male partner & $75(32.2)$ \\
delivery & Other member of the & $156(67)$ \\
& family & \\
& TBA & $2(0.9)$ \\
Distance of health facility from & $\geq 5 \mathrm{~km}$ & 164 \\
home & & $(70.4)$ \\
& $<5 \mathrm{~km}$ & $69(29.6)$ \\
\hline
\end{tabular}

birth preparedness and complication readiness for obstetric referral.

Respondent who walk more than $5 \mathrm{~km}$ from home to nearest health facility to have obstetric care, delivery and PNC services were 0.28 times highly accompanied by male partners compared to those who walk less than 5 $\mathrm{km}$. The finding was consistent with other study done Addis Ababa [16].

Our study also showed that women who had any experience of pregnancy complications were 1.79 times more likely to be accompanied by male partner in birth preparedness and complication readiness for obstetric referral than those who didn't have any signs of complication. The finding was consistent with other study done in Uganda [18]. This may be due to spouses might have been worried more than those whose spouses had not experienced any signs of pregnancy complications.

\section{Conclusions}

In general, male partner's involvement in birth preparedness and complication readiness for obstetric referral in the Sodo Town, Wolaita Zone was low. Antenatal care attending and experiencing of obstetric complication were taking major part in determining male partner's involvement in complication readiness. Health care professionals should involve male partners to attend ANC clinic at each stage and arrange special antenatal care conferences which may increase awareness and practice about complication readiness and plan.

Table 4 Factors associated with male involvement during birth preparedness and complication readiness for obstetric referral at Sodo town in Wolaita zone, 2017

\begin{tabular}{|c|c|c|c|c|c|}
\hline \multirow[t]{2}{*}{ Variables } & \multicolumn{2}{|c|}{ Male involvement } & \multirow[t]{2}{*}{ COR $(95 \% \mathrm{Cl})$} & \multirow[t]{2}{*}{ AOR $(95 \% \mathrm{Cl})$} & \multirow[t]{2}{*}{$P$-value } \\
\hline & Yes & No & & & \\
\hline \multicolumn{6}{|l|}{ Women Educational } \\
\hline Primary and below & $26(20.3 \%)$ & $102(79.68 \%)$ & $0.23(0.09-0.64 \%)$ & $0.94(0.24-3.61)$ & .924 \\
\hline Secondary and above & 55 (52.38\%) & $50(47.61 \%)$ & 1.00 & 1.00 & \\
\hline \multicolumn{6}{|l|}{ Male partner education } \\
\hline Primary and below & 89 (51.44\%) & $84(48.55 \%)$ & $1.60(1.07-2.61)$ & $1.09(0.43-2.74)$ & .858 \\
\hline Secondary and above & $24(40 \%)$ & $36(60 \%)$ & 1.00 & 1.00 & \\
\hline \multicolumn{6}{|l|}{ Women Occupation } \\
\hline Formal employment & $48(85.71 \%)$ & $8(14.28 \%)$ & $1.86(0.51-7.53)$ & $2.48(0.57-10.83)$ & .227 \\
\hline Informal employment & $135(76.3 \%)$ & $42(23.72 \%)$ & 1.00 & 1.00 & \\
\hline \multicolumn{6}{|l|}{ Male partner occupation } \\
\hline Formal employment & $13(16.3 \%)$ & 67 (83.75\%) & $0.09(0.01-0.73)$ & $0.09(0.01-1.04)$ & .054 \\
\hline Informal employment & $105(68.6 \%)$ & $48(31.37 \%)$ & 1.00 & 1.00 & \\
\hline \multicolumn{6}{|l|}{ Distance of health facility } \\
\hline Less than $5 \mathrm{~km}$ & $10(14.5 \%)$ & $59(85.5 \%)$ & $0.23(0.11-0.56)$ & $0.29(0.12-0.72)$ & .008 \\
\hline More than $5 \mathrm{~km}$ & $69(15.76 \%)$ & $95(84.2 \%)$ & 1.00 & 1.00 & \\
\hline \multicolumn{6}{|l|}{ ANC at least once } \\
\hline Yes & $105(46.6 \%)$ & $120(53.33 \%)$ & $2.62(1.52-4.74)$ & $2.9(1.52-5.51)$ & .001 \\
\hline No & $2(25 \%)$ & $6(75 \%)$ & 1.00 & 1.00 & \\
\hline \multicolumn{6}{|l|}{ Experience of complication } \\
\hline Yes & $1(4.76 \%)$ & $20(95.2 \%)$ & $1.63(1.07-2.64)$ & $1.79(1.06-3.04)$ & 0.030 \\
\hline No & $16(7.54 \%)$ & 196 (92.5\%) & 1.00 & 1.00 & \\
\hline
\end{tabular}

Statistically significant at $p<0.05$ 


\section{Abbreviations}

ANC: Antenatal Care; AOR: Adjusted odds ratio; EDHS: Ethiopian Demographic Health Survey; FMOH: Federal Ministry of Health; HEWS: Health Extension Workers; JHPIEGO: John Hopkins Program for International Education in Gynecology Obstetrics; MCH: Maternal and Child Health; MMR: Maternal Mortality Ratio; TBA: Traditional Birth Attendant

\section{Acknowledgments}

We would like to thank Ministry of Education, Wolaita Sodo University and Addis Ababa University for their technical support. Next we would like to acknowledge, Wolaita Sodo thatching and referral hospital, Christian generalized hospital, data collectors, supervisors, study participants and all who gave their hands in the study directly or indirectly without whom the research would not be done.

\section{Authors' contributions}

KP* conceived the original idea and was involved in proposal development, design, and data collection and analysis and in all stages of the research project. NA, BM, and AA participated in proposal development, design, and data analysis and in all stages of the research project. Finally, all authors revised the manuscript and approved the final version.

\section{Funding}

The source of funding for this research was covered by Ministry of Education in collaboration with Wolaita Sodo University for resources and data collection. The funders had no role in study design, analysis, interpretation, decision to publish, or preparation of the manuscript.

\section{Availability of data and materials}

The datasets used and/or analyzed during the current study are available from the corresponding author on reasonable request.

\section{Ethics approval and consent to participate}

The study protocol was approved by the Addis Ababa University, College of Health and Medical Sciences, Institutional Health Research Ethics Review Committee. Official letters of co-operation was written to all hospitals and concerned bodies to obtain their co-operation in facilitating the study. Information on the study was explained to the participants, including the procedures, potential risks and benefits of the study. Informed voluntary written and signed consent were obtained from all respondents prior to the study.

\section{Consent for publication}

Not applicable.

\section{Competing interests}

The authors declare that they have no competing interests.

\section{Author details}

'Department of Midwifery, College of Health and Medical Sciences, Wolaita Sodo University, Wolaita Sodo, Ethiopia. ${ }^{2}$ Department of Nursing, College of Health Science and Medicine, Wolaita Sodo University, Wolaita Sodo,

Ethiopia. ${ }^{3}$ Department of Midwifery, College of Health and Medical Sciences, Addis Ababa University, Addis Ababa, Ethiopia.

Received: 7 December 2017 Accepted: 21 January 2020

Published online: 30 January 2020

\section{References}

1. WHO. Maternal Death Surveillance and Response. Geneva: World Health Organization; 2013

2. WHO, UNICEF, UNFPA TWB and the UNPD. Trends in Maternal Mortality 1990 to 2013. Geneva: World Health Organization; 2013.

3. Central Statistical Agency (CSA) [Ethiopia] and ICF. Ethiopia Demographic and Health Survey 2016. Addis Ababa and Rockville: CSA and ICF; 2016.

4. Assefa Y, Damme WV, Williams OD, et al. Successes and challenges of the millennium development goals in Ethiopia: lessons for the sustainable development goals. BMJ Glob Health. 2017;2:e000318.

5. National Planning Commission, The Federal Democratic Republic of Ethiopia The second growth and transformation plan (GTP II), 2015/16-2019/20. Addis Ababa: NPC; 2016. http://dagethiopia.org/new/docstation/com_content. article/100/gtpii_english_translation_final_june_21_2016.pdf
6. WHO. Trends in maternal mortality : 1990 to 2010. Geneva: World Health Organization; 2012.

7. Mcpherson RA, Khadka N, Moore JM, Sharma M. Are birth-preparedness Programmes effective ? Results from a field trial in Siraha District. Nepal. 2006;24(4):479-88

8. JHPIEGO. Maternal and Neonatal Health (MNH) Program: Birth preparedness and complication readiness. A matrix of shared responsibilities. Maternal and Neonatal Health. 2001:23-31. Available from http://reprolineplus.org/ system/files/resources/bpcr_matrix.pdf.

9. Babalola S, Fatusi A. Determinants of use of maternal health services in Nigeria-looking beyond individual and household factors. BMC Pregn Childbirth. 2009;9(1):43.

10. Iliyasu Z, Abubakar IS, Galadanci HS, Aliyu MH. Birth preparedness, complication readiness and fathers' participation in maternity care in a northern Nigerian community. Afr J Reprod Health. 2010;14(1).

11. WHO, UNICEF, UNFPA WBG and the UNPD. Trends in Maternal Mortality: 1990 to 2015. Geneva: World Health Organization; 2015.

12. Making Pregnancy Safer WHO. Birth and emergency preparedness in antenatal care. Standards for Maternal and Neonatal Care. Geneva: World Health Organization (WHO); 2006.

13. Ibrahim MS, Idris SH, Asuke S, Yahaya SS, Olorukooba AA, Sabitu K. Effect of a behavioral intervention on male involvement in birth preparedness in a rural community in Northern Nigerian. Ann Niger Med. 2014;8(1):20.

14. Roxana C, Del Barco. Monitoring Birth Preparedness and Complication Readiness. Baltimore: Tools and Indicators for Maternal and Newborn Health, JHPIEGO; 2004. Available from https://pdf.usaid.gov/pdf_docs/ pnada619.pdf.

15. Gidey G. Assessment of husbands' participation on birth preparedness and complication readiness in Enderta Woreda, Tigray region, Ethiopia, 2012. J Women's Heal Care. 2014;3(1):1-7 Available from: http://www.omicsgroup. org/journals/assessment-of-husbands-participation-on-birth-preparednessand-complication-readiness-in-enderta-woreda-tigray-region-ethiopia-21670420.1000140.php?aid=24054.

16. Demissie DB, Bulto GA, Terfassa TG. Involvement of male in antenatal care, birth preparedness and complication readinessand associated factors in Ambo town, Ethiopia. J Health Med Nurs. 2016;27(5):14-23.

17. Destaw A. Assessment of husband involvement during Pregnancy and child birth in AkakiKaliti sub-city, Addis Ababa, Ethiopia; 2014.

18. Kakaire O, Kaye DK, Osinde MO. Male involvement in birth preparedness and complication readiness for emergency obstetric referrals in rural Uganda. Reprod Health. 2011;8(1):12.

\section{Publisher's Note}

Springer Nature remains neutral with regard to jurisdictional claims in published maps and institutional affiliations.
Ready to submit your research? Choose BMC and benefit from:
- fast, convenient online submission
- thorough peer review by experienced researchers in your field
- rapid publication on acceptance
- support for research data, including large and complex data types
- gold Open Access which fosters wider collaboration and increased citations
- maximum visibility for your research: over $100 \mathrm{M}$ website views per year
At BMC, research is always in progress.
Learn more biomedcentral.com/submissions 